\section{Predictors of positive treatment response to PTNS in women with overactive bladder}

\author{
Suneetha Rachaneni ${ }^{1 *}$, Doyo Enki ${ }^{2}$, Megan Welstand ${ }^{3}$, \\ Thomasin Heggie ${ }^{4}$ and Anupreet Dua ${ }^{3}$
}

\author{
${ }^{1}$ Shrewsbury and Telford Hospitals NHS Trust, Telford, UK \\ 2University of Nottingham, Nottingham, UK \\ ${ }^{3}$ University Hospitals Plymouth NHS Trust, Plymouth, UK \\ ${ }^{4}$ Northumbria Healthcare Trust, Plymouth, UK
}

\section{Introduction}

Percutaneous tibial nerve stimulation (PTNS) is a noninvasive treatmentfor overactive bladder(OAB). PTNS involves peripheral neuromodulation that uses electrical stimulation to target the spinal cord roots, mainly S3, which controls bladder function. Neuromodulation is postulated to bethe effect of crosssignaling between sympathetic and parasympathetic post ganglionic nerve terminals and synapses, causing alteration of nerve signals involved in the voiding reflex. de Groat, et al. described this neurophysiological process and the neural circuits involved in controlling the lower urinary tract [1]. Stimulation of peripheral nerves and subsequent "cross-talk" at the level of the postganglionic neuroeffector junctions can modulate transmission and facilitate detrusor inhibition [2].

The needle insertion point, situated $4-5 \mathrm{~cm}$ cephalad to the medial malleolus, has previously been acknowledged as a neural access point for the regulation of bladder and pelvic floor function. Klingler reported a reduction of detrusor overactivity [3] and Vandoninck showed an increase of the cystometric capacity and of the threshold of appearance of involuntary detrusor contractions [4].

NICE guidance on Urinary incontinence NG123 (2019) recommends PTNS in refractory OAB patients in a multidisciplinary team setting who are not willing to try botulinum toxin or sacral nerve stimulation. The success rate is an important outcome for evaluating the efficacy of PTNS. In a systematic review, the pooled subjective and objective response rates are $61.4 \%$ and $60.4 \%$, respectively [5]. Efficacy, sustainability of improvement of $\mathrm{OAB}$ and cost-effectiveness are important factors in considering PTNS treatments.

So far, there has been a few little published studies on appropriate patient selection for peripheral neuromodulation.

\section{More Information}

*Address for Correspondence: Suneetha Rachaneni, Shrewsbury and Telford Hospitals NHS Trust, Telford, TF1 6TF, UK, Email: sunilalam@yahoo.com

Submitted: August 21, 2021

Approved: January 17, 2022 Published: January 18, 2022

How to cite this article: Rachaneni S, Enki D, Welstand M, Heggie T, Dua A. Predictors of positive treatment response to PTNS in women with overactive bladder. Clin J Obstet Gynecol. 2022; 5: 001-004.

DOI: 10.29328/journal.cjog.1001097

Copyright License: ๑ 2022 Rachaneni S, et al This is an open access article distributed under the Creative Commons Attribution License, which permits unrestricted use, distribution, and reproduction in any medium, provided the original work is properly cited.

Keywords: Posterior tibial nerve stimulation; Women; Predictors of success; Overactive bladder; Urinary incontinence

W) Check for updates

OPEN ACCESS

They have all reported on different parameters in comorbidities, bladder diaries and urodynamic findings as predictors of treatment success. Daytime urinary frequency and first sensation at bladder filling on cystometry may predict PTNS success [5]. Patients without detrusor overactivity (DO) at baseline were 1.7 times more prone to respond to PTNS than those with urodynamic proven DO [4]. A history of depression/anxiety and severe baseline urgency urinary incontinence were positive predictors of a successful PTNS outcome [6]. We aim to identify any additional patient characteristics who are more likely to respond to this prolonged and relatively expensive treatment and to triage women with $\mathrm{OAB}$ appropriately.

\section{Methods}

We carried out a retrospective study of 103 refractory OAB patients (women who had a trial of atleast two medical treatments and conservative interventions) who underwent PTNS between 2013 to 2017 in our tertiary referral centre, University Hospitals, Plymouth, UK.

Women undergoing PTNS treatment for indications other than $\mathrm{OAB}$ (fecal incontinence, bladder pain, etc.), women discontinuing treatment before completion of 12 cycles were excluded. 
All patients received a weekly stimulation of 30 minutes for a 12 week period. The procedure was as follows: the posterior tibial nerve was located through percutaneous insertion of a needle a few inches above the medial malleolus. After connection to a low voltage stimulator (Cystomedix, Anoka, MN, U.S.A.) and placement of a ground surface electrode on the ipsilateral calcaneus, the tibial nerve was stimulated. This resulted in a tickling sensation in the sole of the foot and flexion of the toes. Subjective outcome was defined by the self-reported symptom improvement or worsening compared with baseline, collected serially at each treatment visit every week based on the patient's memory from the week before.

Patients who had improvement in their $\mathrm{OAB}$ symptoms and requested continuation of treatment after 12 treatment sessions with monthly maintenance therapies due to their subjective success were called 'positive responders'. All women who reported improvement of OAB symptoms after 12 treatment sessions went on to have further monthly top up treatments in this study.

Fifty-three women did not respond to treatment (nonresponders) and the remaining 50 (responders) improved with treatment. Comorbidities, urodynamic parameters, ICIQOAB scores, Charlson Comorbidity Index and bladder diaries in women who did not respond to treatment were compared to those who responded to PTNS.

\section{Statistical analysis}

Demographic data were summarized as mean with standard deviation for continuous variables and as frequencies and percentages for categorical variables in Table 1. Urodynamic data, pre-treatment ICIQ scores and Charlson Comorbidity Index are shown in Table 2.

Chi-square tests for Categorical variables and Student's $\mathrm{t}$ - tests and Mann-Whitney test for continuous variables were used for comparison between groups of non-responders and responders. Multivariable regression models were utilized to identify significant predictors of subjective and objective outcomes. Bivariate and multivariable logistic regression models included both categorical and continuous variables to examine predictors of PTNS therapy success. Statistical analysis was performed with SSPS software. A value of $p<0.05$ was considered statistically significant.

\section{Results}

One hundred and fifty-nine women who underwent PTNS treatment in the Urogynaecology unit at the Plymouth

Table 1: Summary statistics of demographic and clinical characteristics of participants by type of response.

\begin{tabular}{|c|c|c|c|c|}
\hline \multirow{2}{*}{ Characteristics } & \multicolumn{2}{|c|}{ Non-responders } & \multicolumn{2}{|c|}{ Responders } \\
\hline & n used & Value & n used & Value \\
\hline Age in years, mean (SD) & 53 & $64.7(17.8)$ & 50 & $61.4(17.4)$ \\
\hline BMI, mean (SD) & 46 & $30.95(6.7)$ & 41 & $30.67(5.7)$ \\
\hline Parity, median (IQR) (range) & 53 & $2(2)(0-6)$ & 49 & $2(3)(0-6)$ \\
\hline Urinary frequency, mean (SD) & 48 & $10.0(2.98)$ & 46 & $9.8(3.61)$ \\
\hline Nocturia, median (IQR) (range) & 44 & $2(2)(0-8)$ & 48 & $2(2)(0-7)$ \\
\hline Pre-treatment ICIQ scores, mean (SD) & 34 & $11.2(3.08)$ & 39 & $10.2(2.94)$ \\
\hline Charlson index, mean (SD) & 50 & $3.2(2.31)$ & 44 & $3.3(2.33)$ \\
\hline Duration of OAB before presentation (in weeks?), median (IQR) (Range) & 47 & $6(10)(.5-30)$ & 41 & $5(8)(.5-70)$ \\
\hline Had mental health issues, $\mathrm{n}(\%)$ & 53 & 21(39.6) & 47 & $14(29.8)$ \\
\hline Previous treatment, $\mathrm{n}(\%)$ & 52 & $50(96.2)$ & 47 & $45(95.7)$ \\
\hline Bladder training, $\mathrm{n}(\%)$ & 53 & $39(73.6)$ & 50 & $32(64.0)$ \\
\hline Previous Urogynae surgery, $\mathrm{n}(\%)$ & 52 & $25(48.1)$ & 50 & $26(52.0)$ \\
\hline SUI, n (\%) & 53 & $25(47.2)$ & 50 & $22(44.0)$ \\
\hline Interstitial Cystitis/BPS, n (\%) & 52 & $1(1.9)$ & 50 & $2(4.0)$ \\
\hline Prolapse, n (\%) & 53 & $21(39.6)$ & 50 & $15(30.0)$ \\
\hline Recurrent UTI, n (\%) & 53 & $14(26.4)$ & 50 & $6(12.0)$ \\
\hline DO present, $\mathrm{n}(\%)$ & 53 & $31(58.5)$ & 49 & $30(61.2)$ \\
\hline Maximum urgency, median (IQR) (range) & 52 & $3(1)(1-3)$ & 50 & $3(1)(2-3)$ \\
\hline
\end{tabular}

Table 2: Risk factors for predictors of treatment failure. $2 \times 2$ tables for various risk factors for treatment failure along with their $p$ - values

\begin{tabular}{|c|c|c|c|c|}
\hline Risk factors for treatment failure & Present & Non-Respondents & Respondents & $p$ - value \\
\hline \multirow{2}{*}{ Detrusor overactivity } & $\mathrm{N}$ & 22 & 19 & \multirow{2}{*}{0.716} \\
\hline & Y & 31 & 31 & \\
\hline \multirow{2}{*}{ Stress urinary incontinence } & $\mathrm{N}$ & 28 & 28 & \multirow{2}{*}{0.747} \\
\hline & $\mathrm{Y}$ & 25 & 22 & \\
\hline \multirow{2}{*}{ Prolapse } & $\mathrm{N}$ & 32 & 35 & \multirow{2}{*}{0.306} \\
\hline & $\mathrm{Y}$ & 21 & 15 & \\
\hline \multirow{2}{*}{ Recurrent UTI } & $\mathrm{N}$ & 39 & 44 & \multirow{2}{*}{0.065} \\
\hline & $\mathrm{Y}$ & 14 & 6 & \\
\hline \multirow{2}{*}{ Bladder Training } & $\mathrm{N}$ & 14 & 18 & \multirow{2}{*}{0.293} \\
\hline & Y & 39 & 32 & \\
\hline \multirow{2}{*}{ Mental Health } & $\mathrm{N}$ & 32 & 33 & \multirow{2}{*}{0.303} \\
\hline & Y & 21 & 14 & \\
\hline
\end{tabular}


University Hospitals until December 2017 were identified. One hundred and three women satisfied our inclusion/exclusion criteria. All 103 women were included in the analysis. The average age of the participants was 61 years in the responder group and slightly higher at 65 years in the non-responder group. There were no differences in the mean BMI and parity in both groups. The duration of $\mathrm{OAB}$ was longer in the nonresponder group (47 weeks) compared to the responder (41 weeks). The prevalence of mental health conditions and other comorbidities (Charlson Comorbidity Index) were slightly higher in the non-responder group compared to the responders. However, the pre-treatment ICIQ scores were lower in the Non-responder group (34) compared to the Responder group (39). There were no differences in the rates of coexisting prolapse, stress urinary incontinence, interstitial cystitis, bladder diary parameters like urgency severity scores, frequency, nocturia, etc in both groups. Please see Table 1 for demographics and clinical characteristics in the Nonresponders compared to responders. No continuous variable is found to be a significant predictor at the univariate level.

On multiple logistic regression, recurrent UTI was found to be significant at 10\% ( $p=0.071)$; No other variable (categorical or continuous) was found to be significantly associated to the response. Table 2 summarizes the $2 \times 2$ tables for various risk factors for treatment failure along with their $p$ - values.

\section{Discussion}

In our study, we compared the comorbidities, bladder diaries, urodynamic parameters and other coexisting conditions in the group of women who responded to treatment (responders) to the non-responders to identify predictors of successful treatment response. Women with $\mathrm{OAB}$ symptoms and coexisting history of recurrent UTI were found to have reduced response to treatment $(p=0.065)$. There was a trend towards reduced response in women with increased comorbidities, and mental health conditions but the differences were not statistically significant.

Though there was previously published evidence on this study question, none of the previous studies compared the ICIQ-OAB scores, bladder diaries, urodynamic parameters and comorbidities between responders and non-responders to PTNS treatment. Also, there seems to be a huge variation in the predictors studied, the time frame of PTNS treatment and the results presented.

Vandoninck, et al. studied the effect of PTNS on urodynamic parameters before and after the completion of 12 treatment sessions in a small cohort of 46 patients. Similar to the findings in our study, none of the baseline characteristics (cystometric capacity, volume at detrusor instability, detrusor pressure at detrusor instability) proved to be a significant predictor for subjective success. However, the presence of detrusor overactivity and early onset detrusor overactivity (at small bladder capacity were found to be negative predictors of treatment success [4].

Van Balken, et al. showed that poor mental health as measured with low SF-36 Mental component summary is a negative predictor of success of PTNS in a small study of 83 patients [8]. Whether increased severity of mental illness is a negative predictor of treatment success is not clear. There was a similar trend in our study though the difference in the incidence of mental health conditions in the non-responders and responders was not statistically significant.

In a study by Rostaminia, et al. more severe baseline UUI, history of depression/anxiety were independently predictive of treatment success, while more severe nocturia $(>2)$ and severe detrusor over-activity predicted less successful outcomes [7]. A history of previous pelvic floor surgery was not related to treatment failure of PTNS. However, the subjective and objective outcome measures used in this study are not validated. Subjective improvement or worsening was recorded from baseline on a scale of $-100 \%$ to $+100 \%$ over the prior week from the patient's memory. Objective outcomes were based on the patient's memory to quantify the intervoiding interval, nocturia episodes and urgency urinary incontinence (UUI) episode frequency over the prior week. Seven patients had prior sacral neuromodulation and 17 had previous botox treatment suggesting that that study cohort included patients at a more severe end of the spectrum compared to the women in our study. Whether an ongoing effect of these treatments (SNS and Botox) had an impact on the treatment success with PTNS is not known. Also, the study included both men and women and hence it is difficult to draw comparisons with our study.

In another retrospective cohort study of 66 women treatment responders at the end of 12 months of monthly PTNS sessions after the initial weekly treatments for 12 weeks, a history of previous urogynecologic surgery(prolapse or incontinence) was found to be a negative predictor of treatment success [8]. The results of this study needed to be interpreted with caution as 3 patients had previous sacral neuromodulation and 7 had prior botox treatment which might have affected the results. Both of the above studies have conflicted each other with regards to a history of previous urogynaecological surgery history.

Iyer, et al. [10] in a retrospective study identified a direct correlation between the number of PTNS sessions and the response to treatment. The TROOP trial compared PTNS with Botox for refractory OAB. Though patients had improvement in OAB symptoms with both the treatments, patients who received Botox had greater improvement in urgency, frequency, nocturia, and urgency incontinence [11].

Strengths of our study include a relatively large study population, a cohort of only women, comparison of urodynamic parameters between responders and non-responders, 
comorbidities comparison between both the groups, comparison of coexisting urogynaecological conditions like stress urinary incontinence, prolapse and recurrent UTIs and PTNS treatments delivered by nurses highly experienced with PTNS practicing in a single tertiary setting. Additionally, our urodynamics protocol, serial measurement of the patient self-reported outcome at each treatment visit allowed us to analyze a wide range of variables and assess their role in treatment success. Another strength of our study is that we used only validated questionnaires like ICIQ-OAB and bladder diaries.

Limitations of the study are that it is retrospective in nature. We were unable to access data that were not present in the medical record or collected at that time. Our outcome measures were patient reported. We choose to proceed with the study despite some previous evidence as there was a huge variation in the predictors studied and we felt that our experience and results were valuable to practicing urologists/ urogynecologists and our data captured reflected the overall feeling of benefit. Clinic documentation during PTNS therapy sessions by our nursing staff was standardized prior to the time of data capture.

A sufficiently powered prospective randomized controlled trial looking at all the positive and negative predictors identified in the above studies will be able to answer this question appropriately. Results of our study have shown that women with coexisting urinary tract infections are less likely to respond to the PTNS treatment. If confirmed, an algorithm can be developed where only women without severe OAB based on other studies and coexisting recurrent UTI based on our study get offered this treatment.

\section{Conclusion}

Our study has identified a trend towards increased treatment failure in women with a longer duration of $\mathrm{OAB}$, increased comorbidities, increased mental health problems and also those with a coexisting history of recurrent UTI. These findings may help to refine patient selection for PTNS as clinicians continue to understand the most efficient ways in which to target this promising therapy.

\section{References}

1. de Groat WC, Griffiths D, Yoshimura N: Neural control of the lower urinary tract. Comprehensive Physiology. 2015; 5: 327-396. PubMed: https://pubmed.ncbi.nlm.nih.gov/25589273/

2. Hubscher $\mathrm{CH}$, Gupta DS, Brink TS. Convergence and cross talk in urogenital neural circuitries. J Neurophysiol. 2013; 110: 1997-2005. PubMed: https://pubmed.ncbi.nlm.nih.gov/23926033/

3. Klingler HC, Pycha A, Schmidbauer J, Marberger M. Use of peripheral neuromodulation of the $\mathrm{S} 3$ region for treatment of detrusor overactivity: a urodynamic-based study. Urology. 2000; 56: 766-771. PubMed: https://pubmed.ncbi.nlm.nih.gov/11068296/

4. Vandoninck V, van Balken MR, Finazzi Agrò E, Petta F, Micali F, et al. Percutaneous tibial nerve stimulation in the treatment of overactive bladder: urodynamic data. Neurourol Urodyn. 2003; 22: 227-232. PubMed: https://pubmed.ncbi.nlm.nih.gov/12707873/

5. Wang M, Jian Z, Ma Y, Jin X, Li H, et al. Percutaneous tibial nerve stimulation for overactive bladder syndrome: a systematic review and meta-analysis. Int Urogynecol J. 2020; 31: 2457-2471. PubMed: https://pubmed.ncbi.nlm.nih.gov/32681345/

6. Del Río-Gonzalez S, Aragon IM, Castillo E, Milla-España F, Galacho A et al. Percutaneous Tibial Nerve Stimulation Therapy for Overactive Bladder Syndrome: Clinical Effectiveness, Urodynamic, and Durability Evaluation. Urology. 2017; 108: 52-58. PubMed: https://pubmed.ncbi.nlm.nih.gov/28687483/

7. Rostaminia G, Chang C, Pincus JB, Sand PK, Goldberg RP. Predictors of successful percutaneous tibial nerve stimulation (PTNS) in the treatment of overactive bladder syndrome. Int Urogynecol J. 2019; 30 : 1735-1745.

PubMed: https://pubmed.ncbi.nlm.nih.gov/30498931/

8. van Balken MR, Vergunst $\mathrm{H}$, Bemelmans BL. Prognostic factors for successful percutaneous tibial nerve stimulation. Eur Urol. 2006; 49: 360-365

PubMed: https://pubmed.ncbi.nlm.nih.gov/16359781/

9. Pincus J, Rostaminia G, Chang C, Gafni-Kane A, Goldberg RP. Factors associated with overactive bladder symptom improvement after 1 year of monthly percutaneous tibial nerve stimulation therapy. Neurourol Urodynamics. 2019; 38: 1676-1684. PubMed: https://pubmed.ncbi.nlm.nih.gov/31107570/

10. Iyer S, Laus K, Rugino A, Botros C, Lozo S, et al. Subjective and objective responses to PTNS and predictors for success: a retrospective cohort study of percutaneous tibial nerve stimulation for overactive bladder. Int Urogynecol J. 2019; 30: 1253-1259.

PubMed: https://pubmed.ncbi.nlm.nih.gov/30467763/

11. Kopcsay KS, Marczak TD, Jeppson PC, Cameron AP, Khavari R, et al. Treatment of refractory overactive bladder with OnabotulinumtoxinA vs PTNS: TROOP trial. Int Urogynecol J. 2022.

PubMed: https://pubmed.ncbi.nlm.nih.gov/34993598/ 